Z. Klin. Chem. Klin. Biochem.

13. Jg. 1975, S. 117-121

\title{
Vergleiche konventioneller Methoden zur Albumin-, Transferrin- und Coeruloplasmin-Bestimmung im Serum mit immunologischen Referenzmethoden
}

\author{
Von H. Schmidt, H. Ebeling und D. Kraft \\ Aus dem Institut für Klinische Chemie und Klinische Biochemie (Direktor: Prof. Dr. H.-J. Dulce) und der \\ Medizinischen Klinik am Klinikum Steglitz der Freien Universität Berlin
}

(Eingegangen am 13. Januar/21. Februar 1975)

\begin{abstract}
Als bisher üblicher Referenzmethode wird der radialen Immundiffusion die mechanisierte Immunpräzipitation gegenübergestellt. Beide spezifischen Verfahren werden mit je zwei klinisch-chemisch tiblichen Methoden zur Albumin- und Transferrin- und einer enzymatischen Methode zur Cocruloplasmin-Bestimmung verglichen. Dazu werden u. a. dic Korrelationskoeffizienten, die mittleren Regressionsgeraden und die prozentualen Mittelwertsabweichungen von den Mittelwerten der Referenzmethoden erreclinet. Bei Albuminund Cocruloplasmin-Bestimmungen kommen radiale Immundiffusion und mechanisierte Inmunpräzipitation zu gleichen Ergebnissen. Bezüglich der Transferrin-Bestimmungen differicren erstaunlicherwejse beide spezifischen Verfahren. Sowcit an der Richtigkeit der mechanisierten Immunpräzipitation keine Zweifel bestehen, empfehlen wir diesc Methode, da sic schon aus Kosten- und Zeitersparnisgründen überlegen ist.
\end{abstract}

\section{Comparison of conventional methods for the determination of albumin, transferrin and ceruloplasmin in serum with} immunological reference methods

Radial immunodiffusion, which is a presently accepted reference method, was compared with the mechanized immunoprecipitation. Each of these specific methods was compared with two normal clinical-chemical methods for the determination of albumin and transferrin, and an enzymic method for the determination of ceruloplasinin. The correlation coefficients. the mean slope for regression, and the percentage variation of the average values from those of the average values of the reference methods are presented. In the determination of albumin and ceruloplasmin, radial immunodiffusion and mechanized immunoprccipitation give the same results. Surprisingly, these two specific methods give different results in the determination of transferrin. In as far as there is no doubt concerning the accuracy of the mechanized immunoprecipitation, this method is recommended, since it also has the advantage of being less demanding in cost and time.

Mit der Einführung spezifisch-immunologischer Methoden in die Klinische Chemie stellt sich die Frage nach der „Richtigkeit" bisher üblicher klinisch-chemischer Analytik.

An den Beispielen Albumin, Transferrin beziehungsweise totaler Eisenbindungskapazität und Coeruloplasmin werden Methodenvergleiche angestellt und mit sogenannten Referenzmethoden in Beziehung gesetzt.

Für Serumprotein-Bestimmungen galt bisher die einfache radiale Immundiffusions-Methode (1) als Referenzmethode der Wahl. Diesem Verfahren wird als neue Referenzmethode die mechanisierte spezifisch-immunologische Bestimmung mit einer nephelometrischen Meßtechnik gegenübergestellt (2).

\section{Material und Methoden}

Aus Seren, die für etwa drei Monate bei $-20^{\circ} \mathrm{C}$ gelagert waren und von chronisch-hämodialysierten Paticnten stammten, wurden Analysen mit folgenden Verfahren durchgeführt:

1. Mit dem von Ebeling (3) beschriebenen Vorgehen wurden nephelometrisch mit der mechanisierten ImmunpräzipitationsMethode quantitativ Albumin, Transferrin und Cocruloplasmin bestimmt. Es wurden monospezifische Antihuman-Scren der
Firmen BEHRINGWERKE AG, HYLAND und DAKOPATTS verwendet.

2. Mit der von Mancini et al. (1) beschriebenen einfachen radialen Immundiffusions-Methode wurde auf M-Partigen-Immundiffusionsplatten (BEHRINGWERKE AG) ebenfalls quantitativ Albumin, Transferrin und Coeruloplasmin crmittelt.

3. Das Gesamtprotein im Serum wurde nach der von Richterich (4) angegcbenen Methode in der Modifikation von Hoffmann et al. (5) mit Biuretreagenz gemessen.

Dic Scrumeiweiß-Elcktrophoresen wurden im Mikromaßstab auf Celluloseacetat-Folien (SATORIUS) in der BOSKAMP-Kammer ausgeführt und nach Ponceau S-Fürbung im Elphormat (BENDER und HOBEIN) ausgewertet. Aus den Relativprozenten der Albuminfraktion und der Serum-Gesamtproteinkonzentration errechnete sich dic Albuminkonzentration.

4. Albumin wurde außerden quantitativ mit der Bromcresolgrün-Mikromethode nach Schirardin et al. (6) als Farbtest bestimmt.

5. Dic Bestimmung der Serum-kisen-Konzentration $(=\mathrm{Fc})$ mit Bathophenanthrolin und dem TECINICON Autoanalyzer erfolgte in Anjchnung an dic von Zak et al. (7) beschricbenc Methode.

6. Dic latente Eisenbindungskapazität urmittelten wir mit dem auf Ionenaustausch beruhenden Irosorb-59-Test (ABBOTT).

Die Radioaktivitat wurde im Bohrloch-Scintillations-Spektrometer-Zähler (FRIESICKE und HOEPFNER) gemessen.

7. Die totalc Scrumeisenhindungskapazitiit wurde manucll mit ciner Mikromethode in Anlelınung an Führ $(8,9)$ bestimmt. 
8. Als klinisch-chemischen Test zur Coeruloplasminbestimmung benutzten wir den Enzym-Test (DR. HAURY), der die OxydoReduktase-Eigenschaft des Coeruloplasmin durch Umsetzen des Paraphenylendiamin mißt.

Für die immunologischen Methoden wurden zur Kalibrierung beziehungsweise zur Qualitätskontrolle Protein-Standards und Kontrollseren der BEHRINGWERKE AG verwendet. Zur Qualitätskontrolle der kolorimetrischen Methoden und für das Irosorb-Verfahren benutzten wir gleichfalls Kontrollseren der BEHRINGWERKE AG neben Seren der Firma DADE.

\section{Ergebnisse}

Für die Einzelbewertung der benutzten Methoden ermittelten wir u. a. ihre Präzision, zum Methodenvergleich und Abschätzen der Richtigkeit die mittlere Regressionsgerade und die prozentualen Mittelwertsabweichungen zu den Referenzmethoden.

In Tabelle 1 sind die Variationskoeffizienten (=VK) der Präzisionsmessungen der einzelnen Bestimmungsmethoden wiedergegeben. Bei zusammengesetzten Bestimmungen enthält der VK den relativen fortgeleiteten Fehler.

Die Konzentrationsangaben der Präzisionskontrollen geben Aufschluß über die jeweilige Methodenempfindlichkeit und lassen indirekt eine Aussage über den methodenabhängigen Serumbedarf zu.

Um totale Eisenbindungskapazitätswerte mit den Ergebnissen der mechanisierten Immunpräzipitation und der radialen Immundiffusion vergleichen zu können, rechneten wir die Transferrinwerte in Transferrin-Eisenbindungskapazitätswerte um. Hierbei gingen wir davon aus, daß

Tab. 1. Präzision der Bestimmungsmethoden eines Kontrollserums.

\begin{tabular}{|c|c|c|c|}
\hline - & $\mathbf{N}$ & $\begin{array}{l}\text { Konzen- } \\
\text { tration } \\
\text { der Prä- } \\
\text { zisions- } \\
\text { kontrolle }\end{array}$ & VK \% \\
\hline Albumin & & $g / 1$ & \\
\hline $\begin{array}{l}\text { mechanisierte Immunpräzipitation } \\
\text { radiale Immundiffusion } \\
\text { Farbtest } \\
\text { Elektrophorese }\end{array}$ & $\begin{array}{r}12 \\
60 \\
20 \\
5\end{array}$ & $\begin{array}{l}0,0188 \\
3,27 \\
42,0 \\
42,9\end{array}$ & $\begin{array}{l}0,66+++ \\
0,66++ \\
2,28++ \\
2,20+++\end{array}$ \\
\hline Eisenbindungskapazität & & $\mu \mathrm{mol} / 1$ & \\
\hline $\begin{array}{l}\text { mechanisierte Immunpräzipitation } \\
\text { radiale Immundiffusion } \\
\text { latente Eisenbindungskapazität }+\mathrm{Fe} \\
\text { totale Eisenbindungskapazität }\end{array}$ & $\begin{array}{r}15 \\
28 \\
8 \\
20\end{array}$ & $\begin{array}{l}4,315 \\
78,85 \\
59,6 \\
74,7\end{array}$ & $\begin{array}{l}2,16+++ \\
1,00++ \\
7,84+++ \\
4,19++\end{array}$ \\
\hline Coeruloplasmin & & $\begin{array}{l}\mathrm{mg} / \mathrm{l} \text { bzw. } \\
\mathrm{U} / 1\end{array}$ & \\
\hline $\begin{array}{l}\text { mechanisierte Immunpräzipitation } \\
\text { radiale Immundiffusion } \\
\text { enzymatisch }\end{array}$ & $\begin{array}{r}10 \\
8 \\
8\end{array}$ & $\begin{array}{c}4,918 \\
190,0 \\
23,4\end{array}$ & $\begin{array}{l}3,28+++ \\
1,13++ \\
5,48+\end{array}$ \\
\hline
\end{tabular}

ein Transferrin-Molekül bei Eisensättigung zwei Eisenatome $\left(=\mathrm{Fe}^{+++}\right)$bindet und das Molekulargewicht von Transferrin $89000(10)$ beträgt.

Die Ergebnisse der Methodenvergleiche untereinander und mit den jeweiligen Referenzmethoden zeigt

Tabelle 2.

Die graphischen Darstellungen geben nur die Ergebnisse der spezifisch-immunologischen Methodẹnvergleiche wieder. Neben den Meßpunkten und der mittleren Regressionsgeraden zeigen sie auch noch die geforderte oder erwartete ideale Beziehung der Methoden zueinander.

In Tabelle 3 wurden u. a. die Ergebnisse dargestellt, die sich aus der prozentualen Abweichung des jeweiligen Methodenmittelwertes vom Mittelwert der jeweiligen $\mathbf{R e}-$ ferenzmethode errechneten.

Täb. 2. Methodenvergleiche mit Korrelationskoeffizient und mittlerer Regressionsgerade.

\begin{tabular}{|c|c|c|c|c|}
\hline & \multirow[t]{2}{*}{$\mathbf{N}$} & \multirow[t]{2}{*}{$\mathbf{r}$} & \multicolumn{2}{|c|}{$\begin{array}{l}\text { mittlere } \\
\text { Regressionsgeráde } \\
(y=a x \pm b)\end{array}$} \\
\hline & & & $\mathbf{a}$ & $\mathbf{b}$ \\
\hline \multicolumn{5}{|l|}{ Albumin } \\
\hline $\begin{array}{l}\text { RID/AIP } \\
\text { Farbtest/AIP } \\
\text { Elektrophorese/AIP } \\
\text { Farbtest/RID } \\
\text { Elektrophorese/RID } \\
\text { Farbtest/ } \\
\text { Elektrophorese }\end{array}$ & $\begin{array}{l}39 \\
42 \\
39 \\
42 \\
45 \\
45\end{array}$ & $\begin{array}{l}0,935 \\
0,937 \\
0,913 \\
0,975 \\
0,954 \\
0,946\end{array}$ & $\begin{array}{l}1,11 \\
1,08 \\
1,11 \\
0,98 \\
1,02 \\
1,04\end{array}$ & $\begin{array}{l}-\quad 3,64 \\
-\quad 5,56 \\
-\quad 5,33 \\
-\quad 1,49 \\
-\quad 1,98 \\
+\quad 0,30\end{array}$ \\
\hline \multicolumn{5}{|l|}{ Eisenbindungskapazität } \\
\hline $\begin{array}{l}\text { AIP/RID } \\
\text { latente EBK + Fe/AIP } \\
\text { totale EBK/AIP } \\
\text { latente EBK + Fe/RID } \\
\text { totale EBK/RID } \\
\text { latente EBK + Fe/ } \\
\text { totale EBK }\end{array}$ & $\begin{array}{l}40 \\
37 \\
35 \\
34 \\
40 \\
38\end{array}$ & $\begin{array}{l}0,921 \\
0,907 \\
0,938 \\
0,933 \\
0,944 \\
0,948\end{array}$ & $\begin{array}{l}0,95 \\
1,12 \\
0,84 \\
1,30 \\
1,09 \\
1,13\end{array}$ & $\begin{array}{l}+8,75 \\
-18,02 \\
-\quad 3,82 \\
-20,97 \\
-13,29 \\
+\quad 0,63\end{array}$ \\
\hline \multicolumn{5}{|l|}{ Coeruloplasmin } \\
\hline $\begin{array}{l}\text { AIP/RID } \\
\text { enzymatisch/AIP } \\
\text { enzymatisch/RID }\end{array}$ & $\begin{array}{l}37 \\
37 \\
37\end{array}$ & $\begin{array}{l}0,923 \\
0,915 \\
0,882\end{array}$ & $\begin{array}{r}0,99 \\
15,43 \\
1,20 \\
13,14 \\
1,03\end{array}$ & $\begin{array}{l}+9,96 \\
-90,30 \\
-0,70++++ \\
-15,46 \\
-0,20++++\end{array}$ \\
\hline $\begin{array}{l}\text { hier wurde mit } \\
12,82 \text { gerechnet }\end{array}$ & , sie & Texter & rung. & faktor \\
\hline $\begin{array}{l}\text { RID } \\
\text { AIP } \\
\text { latente EBK + Fe } \\
\text { totale EBK }\end{array}$ & $\begin{array}{l}\text { radial } \\
\text { mech } \\
\text { laten } \\
\text { totale }\end{array}$ & $\begin{array}{l}\text { Immund } \\
\text { isierte I } \\
\text { Eisenbir } \\
\text { gisenbin }\end{array}$ & $\begin{array}{l}\text { usion } \\
\text { lunprä } \\
\text { ngska } \\
\text { gskkap }\end{array}$ & $\begin{array}{l}\text { itation } \\
\text { ität }+\mathrm{Fe} \\
\text { ät }\end{array}$ \\
\hline
\end{tabular}

\section{Diskussion}

Für die Richtigkeit aller hier benutzten Referenzmethoden sollte als unabdingbare Voraussetzung gelten, daß es sich um Analysen-Verfahren mit optimaler Antigen-Antikörper-Spezifität und -Qualität handelt. Däbei sollten 
Tab. 3. Mittelwertsabweichungen in Prozent vom Mittelwert dcr Referenz-Methode.

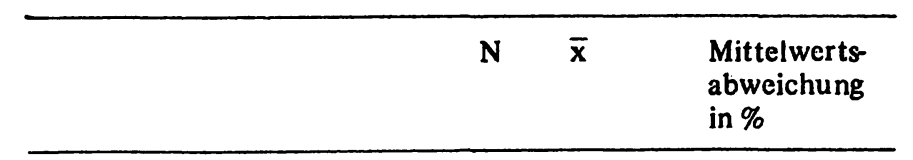

\begin{tabular}{|c|c|c|c|}
\hline Albumin & & $\mathrm{g} / 1$ & \\
\hline $\begin{array}{l}\text { mechanisierte } \\
\text { Immunpräzipitation }\end{array}$ & 40 & 36,3 & \\
\hline $\begin{array}{l}\text { radiale Immundiffusion } \\
\text { Farbtest } \\
\text { Elektrophorese }\end{array}$ & $\begin{array}{l}42 \\
43 \\
43\end{array}$ & $\begin{array}{l}36,2 \\
38,8 \\
37,9\end{array}$ & $\begin{array}{l}-\quad 0,25 \\
+\quad 7,00 \\
+\quad 4,50\end{array}$ \\
\hline $\begin{array}{l}\text { radiale Immundiffusion } \\
\text { mechanisierte }\end{array}$ & 42 & 36,2 & \\
\hline $\begin{array}{l}\text { Immunpräzipitation } \\
\text { Farbtest } \\
\text { Elektrophorese }\end{array}$ & $\begin{array}{l}40 \\
43 \\
43\end{array}$ & $\begin{array}{l}36,3 \\
38,8 \\
37,9\end{array}$ & $\begin{array}{l}+\quad 0,25 \\
+\quad 7,00 \\
+\quad 4,50\end{array}$ \\
\hline Eisenbindungskapazität & & $\mu \mathrm{mol} / 1$ & \\
\hline $\begin{array}{l}\text { mechanisierte } \\
\text { Immunpräzipitation }\end{array}$ & 38 & 54,8 & \\
\hline $\begin{array}{l}\text { radiale Immundiffusion } \\
\text { latente }\end{array}$ & 38 & 62,7 & $+14,40$ \\
\hline $\begin{array}{l}\text { Eisenbindungskapazität }+\mathrm{Fe} \\
\text { totale Eisenbindungskapazität }\end{array}$ & $\begin{array}{l}37 \\
38\end{array}$ & $\begin{array}{l}63,6 \\
70,9\end{array}$ & $\begin{array}{l}+16,10 \\
+29,40\end{array}$ \\
\hline radiale Immundiffusion & 38 & 62,7 & \\
\hline $\begin{array}{l}\text { mechanisierte } \\
\text { Immunpräzipitation } \\
\text { latente }\end{array}$ & 38 & 54,8 & $-12,60$ \\
\hline $\begin{array}{l}\text { Eisenbindungskapazität }+\mathrm{Fe} \\
\text { totale Eisenbindungskapazität }\end{array}$ & $\begin{array}{l}37 \\
38\end{array}$ & $\begin{array}{l}63,6 \\
70,9\end{array}$ & $\begin{array}{l}+1,40 \\
+13,10\end{array}$ \\
\hline Coeruloplasmin & & $\underset{\mathrm{U} / 1}{\mathrm{mg} / 1 \mathrm{bzu}}$ & \\
\hline $\begin{array}{l}\text { mechanisierte } \\
\text { Immunpräzipitation }\end{array}$ & 37 & 349,80 & \\
\hline $\begin{array}{l}\text { radiale Immundiffusion } \\
\text { enzymatisch }\end{array}$ & $\begin{array}{l}37 \\
37\end{array}$ & $\begin{array}{r}354,96 \\
27,73\end{array}$ & $\begin{array}{l}+1,48 \\
+1,65++++\end{array}$ \\
\hline radiale Immundiffusion & 37 & 354,96 & \\
\hline $\begin{array}{l}\text { mechanisierte } \\
\text { Immunpräzipitation } \\
\text { enzymatisch }\end{array}$ & $\begin{array}{l}37 \\
37\end{array}$ & $\begin{array}{r}349,80 \\
27,73\end{array}$ & $\begin{array}{l}-1,45 \\
+0,14++++\end{array}$ \\
\hline
\end{tabular}

+++ hier wurde mit dem ermittelten Umrechnungsfaktor 12,82 gerechnet, siehe Texterläuterung.

stabile, qualitativ ausreichend konzentrierte, hoch-monospezifische Antiseren und vor allem bei exakten quantitativen Analysen Protein-Eichstandards von besonders hoher Qualität, Stabilität und Einwaage zur Verfügung stehen. Mit Erfüllen oder Nichterfüllen dieser Voraussetzungen beantwortet sich die Frage nach der Richtigkeit der Methoden von selbst.

Wie die Albumin-Methodenvergleiche zeigen, erbringen die einfache radiale Immundiffusions- und die mechanisierte nephelometrische Immunpräzipitations-Methode gleiche Ergebnisse. Bei gleich guter Präzision sind somit beide spezifischen Albumin-Bestimmungsmethoden als gleichwertige Referenzmethoden anzusehen. Aus Kostenund Zeitersparnisgründen geben wir heute jedoch der mechanisierten Immunpräzipitation den Vorzug $(3,11)$. Im Vergleich dazu ergeben beide unspezifischen Albu-
min-Bestimmungsmethoden, das Farbstoffbindungsverfahren mit Bromcresolgrün und die elektrophoretische Auftrennung und Quantifizierung der Relativprozente durch Gesamteiweißbestimmungen, zu hohe Werte. Offensichtlich ist die Anfärbbarkeit des Albumin nach der Serumelektrophorese mit Ponceau S höher als die der andern Protein-Fraktionen. Dies ist sicherlich u. a. dadurch bedingt, daß die sogenannte Albumin-Bande keine protein-chemisch einheitliche Fraktion ist und somit andere Proteine, neben der unterschiedlichen ProteinFarbstoffaffinität, mitgemessen werden (12). Die Bromcresolgrün-Methode erfaßt ebenfalls nicht spezifisch nur Albumin, da auch andere Proteine anscheinend mitreagieren und höhere Albuminwerte vortäuschen (11). Jedoch korrelieren beide letztgenannten Verfahren gut miteinander in Bestätigung von Schirardin et al. (6) und Booij (12).

Um die beiden spezifischen immunologischen Transferrin-Bestimmungsmethoden mit den unspezifischen, den Serum-Eisenbindungskapazitäts-Methoden, vergleichen zu können, haben wir in Anlehnung an Haeckel et al. (13) die Transferrin-Konzentrationen als Transferrin-Eisenbindungskapazität ausgedrückt. Wie unsere Ergebnisse erneut bestätigen, messen die unspezifischen totalen Serum-Eisenbindungskapazitätsmethoden, die Irosorb-59- plus Serum-Eisen-Methode und vor allem die Methode nach Führ höhere Werte im Vergleich zu den spezifischen Methoden. Daß eine Diskrepanz zwischen totaler und der Transferrin-Eisenbindungskapazität besteht, fanden u. a. Stojceski et al. (14) und Scuro et al. (15). Van der Heul et al. (16) konnten zeigen, daß in die totale Serum-Eisenbindungskapazität außer Transferrin auch andere eisenbindende Serumkomponenten mit eingehen. Schon deshalb ist eine Bestimmung der totalen Eisenbindungskapazität mit einer Transferrin-Bestimmung nicht mehr gleichzusetzen (13, 17).

Beim Vergleich der beiden spezifischen Methoden miteinander fällt entgegen unsern eigenen und den Erwartungen anderer Autoren (13) auf, daß die bisher übliche Referenzmethode für Transferrin, die einfache radiale Immundiffusion, höhere Werte gegenüber der mechanisierten Präzipitations-Methode ergibt. Der Mittelwert lag bei der Immundiffusion um etwa 14 Prozent über dem der Nephelometrie. Dieser Unterschied ist mit dem t-Test mit $p<0,025$ wahrscheinlich signifikant.

Die Frage nach dem ursächlichen Zusammenhang dieser erstaunlichen Diskrepanz zwischen beiden Methoden konnte bisher von uns noch nicht geklärt werden. $\mathrm{Zu}$ untersuchen wäre, ob das unterschiedliche physikalischchemische Meßprinzip hier divergente Ergebnisse erbracht hat oder ob die radiale Immundiffusion auf Grund anderer höherer Konzentrationsverhältnisse als bei der Nephelometrie in der Antigen-Antikörper-Reaktion einen „unspezifischen“ Anteil miterfaßt und damit zu höheren Werten kommt. Es sei darauf hingewiesen, 
daß bei beiden Methoden die gleichen Antigen-Standards verwendet wurden, und man sollte annehmen dürfen, daß die Qualität und Spezifität der PartigenplattenAntikörper denen der Antiseren in den Fläschchen von der gleichen Firma entsprachen.

Solange nicht eindeutig geklärt ist, welche der beiden „spezifischen“ Methoden das richtige Ergebnis erbringt, fällt es schwer, einer der beiden Methoden das Prädikat „Referenz"-Methode zuzusprechen.

Beim Coeruloplasmin-Methodenvergleich zeigt sich, daß Bestimmungen über die radiale Immundiffusion und die nephelometrische Immunpräzipitation nur geringfügig differente Werte ergeben. Somit sind wie beim Albumin diese beiden Methoden gleichwertige Referenzmethoden.

Mit der Oxydo-Reduktase-Aktivitätsbestimmung des Coeruloplasmin erhielten wir in dieser Arbeit mit den Referenzmethoden gut korrelierende, jedoch gegenüber den in der Literatur $(18,19)$ angegebenen Vergleichen von $\mathrm{mg} / \mathrm{l} \mathrm{zu} \mathrm{U} / \mathrm{l}$ wie $10,4 / 1$ und $10 / 1$ hier Werte von $\mathrm{mg}$ zu U wie $12,82 \mathrm{zu} 1$. Wie bei Haralambie (19) ergab auch unser Coeruloplasmin-Protein-Standard enzymkinetisch niedrigere Werte gegenüber den immunologisch ermittelten bei Annahmè èines Verhältnisses von $10 / 1 \mathrm{mg} / 1$ immunologisch bestimmt zu U/l enzymkinetisch ermittelt. Die gute Übereinstimmung von 10/1 von immunologisch und enzymatisch ermittelten Werten erhielt Haralambie nur bei Verwendung frischer Seren für die enzymkinetische Methode (19). Trotz beschriebener Enzymaktivitätsverluste (vgl. 1. c. (19)) bleibt die immunologische Aktivität des Coeruloplasmin erhalten (19). Unsere für die Bestimmung benutzten Seren waren sämtlichst für etwa drei Monate bei $-20^{\circ} \mathrm{C}$ eingefroren und ergaben etwa den gleichen Aktivitätsunterschied immunologisch zu enzymatisch wie der benutzte

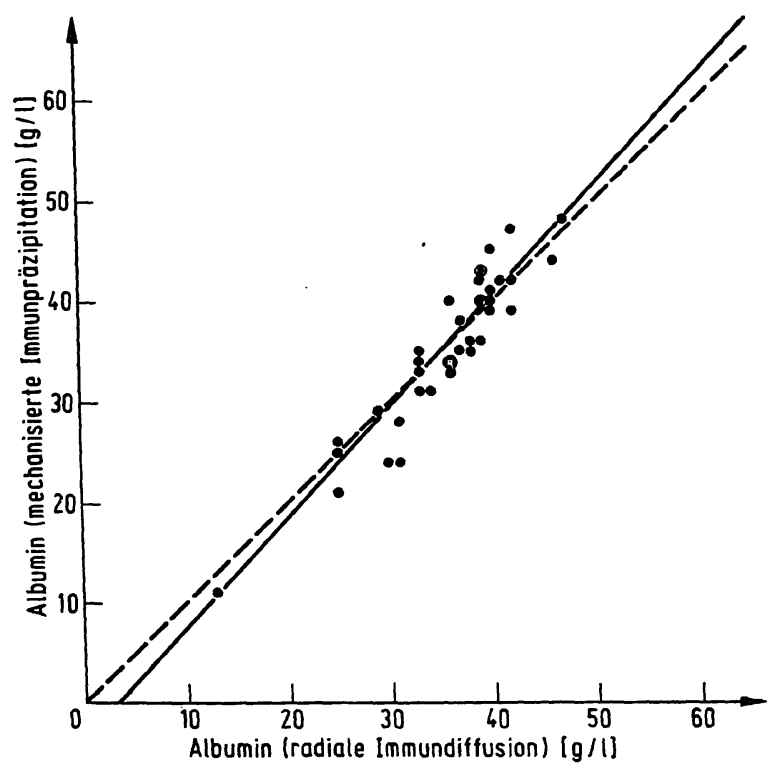

Abb. 1. Methodenvergleich: Albumin mittlere Regressionsgerade,
$-\ldots-$ Idealkurve. lyophilisierte Coeruloplasmin-Standard der BEHRINGWERKE AG mit $300 \mathrm{mg} / 1 \mathrm{zu} \mathrm{23,4} \mathrm{U/1} \mathrm{(siehe} \mathrm{Tab.} \mathrm{3).}$

Das Verhältnis, welches sich aus dem auf Gewichtsbasis standardisierten Coeruloplasmin zu der von uns gemessenen Enzymaktivität ergab, wurde in Tabelle 3 als Umrechnungsfaktor für die prozentuale Mittelwertsabweichung der enzymatischen Methode im Vergleich zu den Referenz-Methoden benutzt.

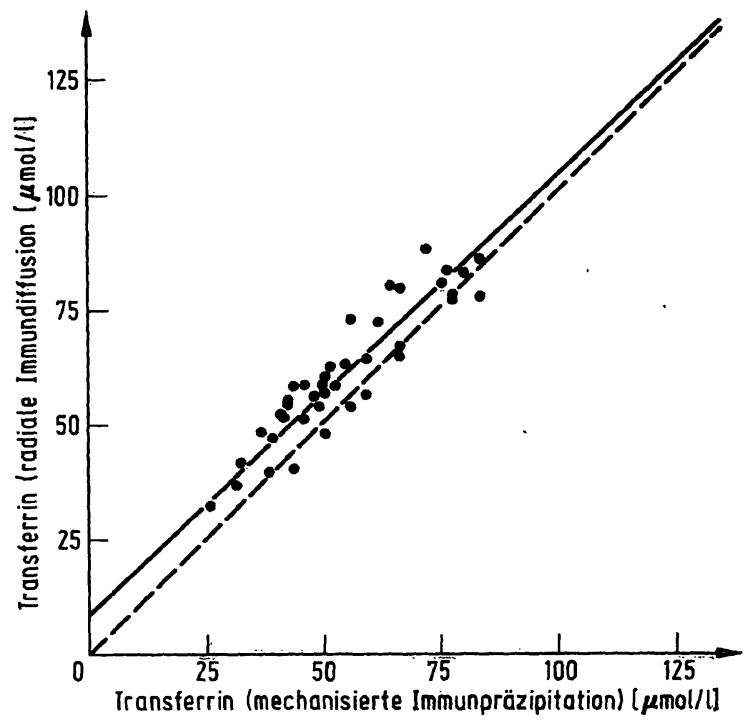

Abb. 2. Methodenvergleich: Transferrin

Die Konzentrationen sind in Transferrin-Eisenbindungskapazitätswerten ausgedrückt.

- mittlere Regressionsgèrade, -_- Idealkurve.

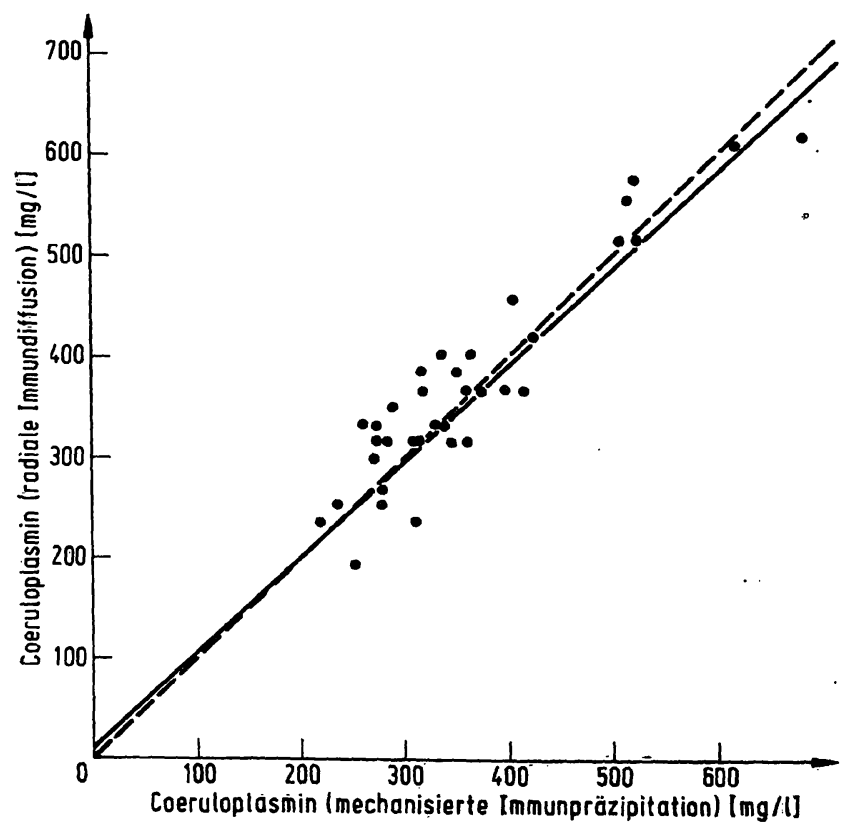

Abb. 3. Methodenvergleich: Coeruloplasmin - mittlere Regressionsgerade, - - Idealkurve. 
Da man offensichtlich bei den beiden spezifischen immunologischen Methoden mit einem immunologischen Aktivitätsverlust nicht zu rechnen hat, im Gegensatz zu der enzymkinetischen Coeruloplasmin-Bestimmung, sind immunologische Werte verläßlicher und reproduzierbarer.

Meßwerte, die mit spezifischen quantitativen AnalysenVerfahren erstellt werden, geben dem Arzt eine sicherere Aussage. Darum ist es notwendig, die bisher noch meist üblichen unspezifischen Verfahren in der Klinischen Chemie zu Gunsten spezifischerer Verfahren zu ersetzen.

Wir empfehlen hier, soweit die „Richtigkeit“ der Methoden keine Zweifel aufkommen läßt, zur Serumprotein- bestimmung die mechanisierte Immunpräzipitation. Wenn wir voraussetzen dürfen, daß heute im Rahmen der allgemeinen Automatisierung Klinik-Laboratorien einen entsprechenden Gerätefundus einschließlich der Durchflußfluoronephelometer (TURNER oder TECHNICON) besitzen, ist die Methode der mechanisierten Immunpräzipitation der Immundiffusion aus Reagenzienkosten- und Zeitersparnisgründen überlegen und, was die Reproduzierbarkeit der Ergebnisse anbelangt, nicht unterlegen.

\section{Danksagung}

Den Firmen ABBOTT, BEHRINGWERKE AG, DAKOPATTS und HYLAND danken wir für die Überlassung von Testpackungen.

\section{Literatur}

1. Mancini, G., Carbonara, A. O. \& Heremans, J. F. (1963), Immunochemistry 2, 235-254.

2. Eckman, I., Robbins, J. B., Van Den Hamer, C. J. A. Lentz, J. \& Scheinberg, I. H. (1970), Clin. Chem. 16, 558561.

3. Ebeling, H. (1973), diese Z. 11, 209-214.

4. Richterich, R. (1971), Klinische Chemie, Theorie und Praxis, 3. Aufl. S. 305-309, S. Karger, Basel.

5. Hoffmann, J. P. \& Richterich, R. (1970), diese Z. 8, 595598.

6. Schirardin, H. \& Ney, J. (1972), diese Z. 10, 336-343.

7. Zak, B. \& Epstein, E. (1965), Clin. Chem. 11, 641-644.

8. Führ, J. (1965), Med. Monatsschrift, 19, 281-283.

9. Führ, J. \& Stary, E. (1970), Technicon Symposium, Sonderdruck R 874.

10. Bambach, M. N. (1966), Klin. Wochenschr. 44, 12761284.
11. Wegfahrt, P. F., Fish, M. B., Aldana, F. B. \& Aronson, S. B. (1971), Technicon International Congress 1970, Vol. 1, Clinical, S. 21-24, Thurman Associates, USA.

12. Booij, J. (1972), Clin. Chim. Acta 38, 355-362.

13. Haeckel, R., Haindl, H., Hultsch, E., Mariss, P. \& Oellerich, M. (1973), diese Z. 11, 529-534.

14. Stojceski, T. K., Malpas, J. S. \& Witts, L. J. (1965), J. Clin. Pathol. 18, 446-452.

15. Scuro, L. A., Dobrilla, G., Lo Cascio, V., Bosello, O., D'Andrea, F. \& Innecco, A. (1972), Acta Hep.-Gastroenterol. $19,90-98$.

16. Van Der Heul, C., Van Eijk, H. G., Wiltink, W. F. \& Leijnse, B. (1972), Clin. Chim. Acta 38, 347-353.

17. Koch, C.-D. \& Ritter, U. (1974), Diagnostik 7, 766-770.

18. Jérôme, H. \& Girault, M. (1969), Ann. Biol. Clin., 27, 371 -385.

19. Haralambie, G. (1969), diese Z. 7, 352-355.
Dr. Herwig Ebeling Institut für Klinische Chemie und Klinische Biochemie Klinikum Steglitz der Freien Universität Berlin 1 Berlin 45,

Hindenburgdamm 30 
. 